R U D O L F A G T N E R

\title{
CIAO TRIESTE - EIN NACHRUF AUF DAS ÖSTERREICHISCHE GENERALKONSULAT UND DIE AUSSENHANDELSSTELLE TRIEST
}

1379 stellte der Graf von Tibein seine Grafschaft unter den Schutz der Erzherzöge von Österreich, um sich vor den Übergriffen des benachbarten Bischofs von Aquileia und der mächtigen Republik Venedig zu schützen - wodurch die Habsburger bei Duino erstmals einen Adriazugang erhielten. Das Gebiet der heutigen Stadt Triest begab sich 1382 unter den Schutz der Habsburger. Jahrhundertelang blieb Triest ein unbedeutender Ort, an eine österreichische Seefahrt in der Adria war nicht zu denken, da hier die venezianische Flagge dominierte. Nachdem die Republik Venedig 1716 ihre Stützpunkte auf der Peloponnes an das Osmanische Reich verloren hatte, musste sie sich mit den Habsburgern ins Einvernehmen setzen. 1717 anerkannte Venedig erstmals die freie Schifffahrt in der Adria. In der Folge erklärte Kaiser Karl VI. am 15. März 1719 Triest und Fiume zu Freihäfen, und am 22. Mai 1719 wurde in Triest die „Privilegierte Orientalische Compagnie“ gegründet. In den folgenden zwei Jahrhunderten entwickelte sich Triest zum wichtigsten Hafen der habsburgischen Erbländer. 1748 errichtete Maria Theresia in Triest eine „Handelsintendanza“, die dem 1746 errichteten Kommerzdirektorium in Wien unterstand. Diese „Intendanza“ wurde 1776 aufgelöst und durch das k. k. küstenländische Gubernium ersetzt. Der Intendant bzw. der Gouverneur von Triest war auch für den Handel und die Konsulate im Westen - damals als „Ponente“ bezeichnet - zuständig.

1837 wurde in Triest unter maßgeblicher Beteiligung des Hauses Rothschild die Dampfschifffahrtsgesellschaft des Österreichischen Lloyd gegründet, die ihre Aktivitäten zunächst auf das östliche Mittelmeer und die Adria konzentrierte.

Am 30. Januar 1850 geruhte S. k. k. Majestät die Errichtung einer eigenen Reichsbehörde zu genehmigen, welche unter der Bezeichnung CentralSeebehörde (Governo centrale marittimo) ihren Amtssitz in Triest haben wird. In der Folge entwickelte sich Triest, nicht zuletzt dank des Österreichischen Lloyd, aber auch der „Austro-Americana“ zum Zentrum der 
österreichischen Handels- und Personenschifffahrt, wozu nicht zuletzt die Eröffnung des Suezkanals im November 1869 beitrug. Durch Triest und den „Österreichischen Lloyd“ wurde Österreich mit der Levante, und durch den Suezkanal nun auch - unter Wegfall tausender Seemeilen - mit Indien, Ostasien und Afrika verbunden, während die der Firma Schenker gehörende „Austro-Americana“ den Linienverkehr mit Nord- und Südamerika übernahm.

Im November 1918 fiel Triest an Italien. Um den Nachschub dringend benötigter Lebensmittel nach Österreich via Triest zu beschleunigen, bestand bald nach Abschluss des Staatsvertrags von St. Germain im September 1919 großes Interesse an einer österreichischen Präsenz in Triest. Am 16. Februar 1920 - d. h. fünf Monate vor Inkrafttreten des Staatsvertrags von St. Germain am 16. Juli 1920 - gab Italien seine Zustimmung zur Anwesenheit eines de facto Generalkonsuls der Republik Österreich. Zunächst wurde Konsularkanzleirat Winkler zur Anmietung von Amtsräumen für das neue Generalkonsulat nach Triest entsandt. Dieser kam streikhalber erst Montag an und mietete für die Zeit vom 1. Mai bis zum 19. September 1920 Amtsräume in der deutschen Schule in der Via San Lazzaro $19 \mathrm{an}^{1}$.

Am 30. April 1920 fand sich das Staatsamt für Äußeres bestimmt, Remi Kwiatkowski mit der Errichtung und Leitung des ÖGK in Triest zu betrauen; dieser traf am 8. Mai 1920 in Triest ein, womit die konsularische Präsenz Österreichs in Italien begann. Das neue Amt nahm seine Tätigkeit am 1. Juni 1920 auf - und hatte reichlich Arbeit: in der ersten JuniHälfte wurden 20 Pässe ausgestellt, 21 verlängert, 1204 Sichtvermerke erteilt und 11 andere Amtshandlungen vorgenommen; man nahm Gebühren in der Höhe von 22.082,25 Lire ein.

Erhebliche Arbeit verursachten Generalkonsul Kwiatkowski die Rückwanderer aus Übersee, die in ihre alte Heimat zurückkehren wollten: $D a$ sowohl seitens der lokalen Polizeibehörde wie auch seitens der hiefür in Betracht kommenden Konsularvertretungen die aus Amerika hier einlangenden Rückwanderer an Bord abgefertigt werden, um deren zeitraubendes und umständliches Herumziehen von Amt zu Amt zu vermeiden, habe ich mich gezwungen gesehen, der hier herrschenden Praxis Rechnung zu tragen und sende je nach Bedarf einen oder zwei der h. a. zugeteilten Beamten an Bord jener Rückwandererschiffe, um die erforderlichen Ein- beziehungsweise Durchreisevisa zu erteilen. Mit Rücksicht auf die große Zahl der Rückwan-

\footnotetext{
${ }^{1}$ Österreichisches Staatsarchiv, Archiv der Republik (AdR), NAR, F 8, K 301, Tel. Winkler, Triest 26. April 1920.
} 
derer kommt es des öfteren vor, dass sich derartige Amtshandlungen an Bord der Schiffe auch bis in den nächsten Tag hinein ausdehnen ${ }^{2}$.

Am 15. September 1920 lief der Mietvertrag ab und Kwiatkowski musste sich Gedanken über die Zukunft des Generalkonsulats machen: Die Wohnungsnot in Triest ist heute in Folge des andauernden militärischen Nachschubs und der Niederlassung von neuen Banken und anderen offiziellen Stellen eine derartige, daß ich trotz Unterstützung des Generalkommissariates bis jetzt weder geeignete Kanzleiräume noch eine Wohnung für mich finden konnte. Durch den Umstand, daß die deutsche Schule, woselbst unsere Amtsräume untergebracht sind, nur eine beschränkte Anzahl von Klassen eröffnet, ist es für uns möglich, in den bisherigen Räumlichkeiten zu bleiben ${ }^{3}$. Kwiatkowski verlängerte den Mietvertrag um weitere sechs Monate.

Im August 1922 übersiedelte er in neue Amtsräume in der Via Ghega 8; das Amt bestand aus 6 stanze, 2 camerini, cucina e cantina ora occupata dalla Società austriaca per l'importazione di oli, semi e foraggi; die jährliche Miete betrug 7287,90 Lire.

Am 23. Dezember 1931 beschloss der Ministerrat die Umwandlung des GK Triest in ein Honoraramt und die Ernennung des Kaufmannes Heinrich Wagner, Chemikalienhändler en gros, zum Honorar-Generalkonsul, weil sich zur Erreichung der dem Außendienst auferlegten sehr weitgehenden Budgetabstriche das Bundeskanzleramt-Auswärtige Angelegenheiten genötigt gesehen hat ... eine Reihe von Dienstposten und auch ganze Ämter mit 1. Jänner 1932 aufzulassen. Wagner wurde als Generalkonsul II. Klasse Karl Nedwed beigegeben, weiters wurden ihm 12.000 Lire Konsulargebühren überlassen, und 24.000 Lire jährlich als Dienstentgelt bewilligt, was die geplante Ersparnis stark reduzierte. Das Amt befand sich von 1932 bis 1938 in der Via Benvenuto Cellini 3.

1934 war eine fühlbare Intensivierung unseres Wirtschaftsverkehrs über Triest eingetreten. Eine weitere Hebung desselben unter tunlichster amtlicher Förderung liegt in dem Bestreben sowohl der Bundesregierung als auch der italienischen Regierung. Unsere Interessenvertretung in Triest ist daher schon jetzt und wird späterhin in gesteigertem Maße vor Aufgaben gestellt, denen sie mit dem gegenwärtigen unzulänglichen Personalstand und unter einer bloß ehrenamtlichen Leitung nicht in wünschenswerter Weise nachzukommen vermag. Auch ist es aus außenpolitischen Gründen empfehlenswert, unserer Interessenvertretung an diesem Ort den Charakter einer vollgültigen

${ }^{2}$ AdR, NAR, F 8, K 301, GK Triest Z1. 751/A vom 9. Aug. 1920.

${ }^{3}$ AdR, NAR, F 8, K 301, GK Triest 1306/A vom 14. Sept. 1920. 
amtlichen Vertretung zu verleihen ${ }^{4}$. Am 9. März 1934 beschloss der Ministerrat die Wiedererrichtung eines effektiven Generalkonsulats in Triest.

Am 23. April 1934 konnte man erstmals in der Zeitung vom österreichischen Generalkonsulat in Triest lesen: ... steht im diplomatischen Außendienste Österreichs eine bedeutungsvolle Neuerung bevor. Bereits in nächster Zeit wird in Triest ein österreichisches Generalkonsulat errichtet werden. Diese Maßnahme steht in engstem Zusammenhang mit der seit langem zielbewußt verfolgten Handelspolitik, die jede Gelegenheit benützt, die schon so erfolgreich angebahnte Erweiterung unserer Handelsbeziehungen ausgreifender zu gestalten. Diese Bestrebungen haben bekanntlich durch die im März unterzeichneten römischen Abkommen zwischen Österreich, Italien und Ungarn mächtigste Förderung erfahren und werden durch die ... gegenwärtig in Rom geführten und hinsichtlich ihres Abschlusses mit 15. Mai terminisierten Verhandlungen in fühlbaren Ergebnissen ihren Ausdruck finden. Im Hinblick auf diesen Ausbau der österreichischen Handelsbeziehungen kommt natürlich dem Triester Hafen besondere Bedeutung zu, daher steht die Errichtung einer Österreich zukommenden Freihafenzone in Triest im Vordergrunde der Erwägungen und Beratungen. Wieweit die diesbezüglichen Vorarbeiten schon gediehen sind, geht daraus hervor, daß nunmehr schon in allernächster Zeit ein österreichisches Generalkonsulat in Triest errichtet werden wird, das einen neuen Stützpunkt des diplomatischen Außendienstes Österreichs darstellen wird ${ }^{5}$.

Am 15. Mai 1934 hatte die Bundesregierung den geeigneten Kandidaten bestimmt: den Tiroler Heimwehrführer und Bundeskommissär für Propaganda, Dr. Richard Steidle, der am 18. Juni 1934 seinen Dienst als Generalkonsul in Triest antrat.

Mit dem Anschluss übernahm das deutsche Konsulat in der Via della Geppa 2 die Agenden des österreichischen Generalkonsulats. Steidle wurde nach Wien einberufen, am 13. März 1938 in Wien verhaftet und am 30. August 1940 im KZ Buchenwald ,,auf der Flucht" erschossen; er war das einzige Todesopfer des NS-Regimes unter Österreichs Diplomaten der Ersten Republik ${ }^{6}$.

Die Bundeskammer der gewerblichen Wirtschaft - die heutige Wirtschaftskammer Österreich - errichtete zur Förderung der österreichi-

${ }^{4}$ AdR, NAR, F 8, K 301, BKA/AA 132.636-13 pers vom 7. März 1934.

${ }_{5}^{5}$ Wiener Montagblatt, 23. April 1934, S. 1.

${ }^{6}$ Eine in der Einfahrt des Palais Ballhausplatz 2 angebrachte Marmortafel zum Gedenken an die Opfer des Nationalsozialismus unter den Angehörigen des Österreichischen Auswärtigen Dienstes erweckt in dieser Hinsicht einen nicht zutreffenden Eindruck. 
schen Exporte mit 1. Januar 1948 eine Außenhandelsstelle („Rappresentanza Commerciale Austriaca") in Triest, die in der Via Mazzini 35 Quartier bezog. Zur Betreuung der österreichischen Kolonie und Wahrnehmung wirtschaftlicher Interessen trat am 8. April 1948 Otto Hausleitner als Leiter der „Paßstelle Triest“" seinen Dienst an; die Stadt war - mit Istrien - durch die Hinterlegung der letzten Ratifikationsurkunde zum Friedensvertrag mit Italien ab 15. September 1947 Teil des „Freien Territoriums Triest". Die Passstelle ${ }^{7}$ befand sich ab 20. April 1948 in der Contrada del Corso 7 und übersiedelte am 15. Dezember 1948 in die Via Santa Catarina 7 .

Im September 1953 - nach der Teilung des Territoriums in eine A- und eine B-Zone - wurde die Passstelle in eine „Verbindungsstelle zur Alliierten Militärregierung in Triest" umgewandelt, zu deren Leiter Generalkonsul Dr. Schullern bestellt wurde. Am 11. Februar 1954 teilte Schullern im „Rundschreiben Nr. 4“ mit, dass sich nunmehr der Verein der Österreicher in Triest gebildet habe, der vorläufig ebenfalls in der Via Santa Catarina 7 seinen Sitz haben sollte.

Ab 5. Januar 1955 führte das Amt in Triest die Bezeichnung „Amt des Vertreters der Österr. Bundesregierung“; Amt und Residenz befanden sich ab 20. Oktober 1955 bis 1979 in einem um 1890 errichteten, oberhalb des Doms von San Giusto liegenden, heute nicht mehr bestehenden Hause des Igino Cociancich in der Via dei Navali 23. Am 25. April 1956 genehmigte der Ministerrat die Umwandlung der „Verbindungsstelle“ in ein Generalkonsulat Triest. Im selben Jahr trat das Abkommen über die Benützung des Hafens von Triest in Kraft, der - wie schon vor 1918 - zum Heimathafen österreichischer Hochseeschiffe wurde; so zeigte sich die rot-weiß-rote Fahne wieder auf den Weltmeeren.

Im Jahre 1967 beschlossen ÖMV, Shell, Mobil, Esso, Agip, Total und BP die gemeinsame Errichtung der Adria-Wien-Pipeline von Triest nach Schwechat. Nach deren Fertigstellung 1970 stieg die Bedeutung Triests für Österreich weiter an - und damit auch die des dortigen Generalkonsulats.

Im Jahre 1979 wurde die Villa in der Via dei Navali 23 aufgegeben. Für die Kanzlei des Generalkonsulats wurde in der Via Fabio Filzi 1 eine große Wohnung angemietet, und als Residenz des Generalkonsuls erwarb Österreich um 600.000 Schweizer Franken die „Villa Metzger“ in der Via Muratori $2 / 2$, samt $5047 \mathrm{~m}^{2}$ Garten.

${ }^{7}$ Die Passstelle im „Freien Territorium Triest“ entsprach den ebenfalls ab Januar 1948 in den drei westlichen Besatzungszonen in Deutschland eingerichteten „Verbindungsstellen“ in Baden-Baden, München, Frankfurt, Düsseldorf und Hamburg, deren Leiter bei den alliierten Befehlshabern akkreditiert waren. 
Im Jahre 1996 erfuhr die österreichische Präsenz in Triest eine erste Verminderung - die Außenhandelstelle, die sich damals in der Via Dante Alighieri 5 befand, wurde nach Padua verlegt. Am 1. Mai 2003 wurde schließlich auch das Österreichische Generalkonsulat Triest geschlossen, angeblich, weil die Kosten des Generalkonsulats in keinem Verhältnis zu dessen Nutzen standen - was allerdings auch bei einer Reihe von Botschaften, welche die Republik an ,exotischen' Plätzen unterhält, der Fall ist. Bei genauerer Betrachtung fällt auf, dass in früheren Jahren viele Diplomaten ihren ersten Posten als Amtsleiter in Triest erhielten und ihre Karrieren dort begannen; in den letzten zehn Jahren der Geschichte des Generalkonsulats hatte sich dies geändert: die letzten vier Generalkonsuln waren alle zuvor Botschafter gewesen und verabschiedeten sich von Triest aus in die Pension. Die endgültige Liquidierung des Generalkonsulats erfolgte mit 31. August 2003; die Betreuung seines bisherigen Konsularbezirkes erfolgt seit September 2003 durch das Österreichische Generalkonsulat Mailand. Die Errichtung eines österreichischen Honorarkonsulats in Triest ist derzeit in Vorbereitung.

Es kann nur als Ironie der Geschichte bezeichnet werden, dass Österreich die Schließung des Österreichischen Generalkonsulats Triest auf den Tag genau ein Jahr vor dem Beitritt der Republik Slowenien zur Europäischen Union vornahm, durch den Triest erstmals seit 1945 wieder sein Hinterland zurückerhielt.

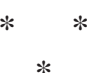

\section{Österreichs Generalkonsulat / Honorargeneralkonsulat / Passstelle / Verbindungsstelle/ Vertreter der Bundesregierung / Generalkonsulat / Honorarkonsulat Triest Die LeITER SEIT 1920}

Remi Kwiatkowski GK 8. Mai 1920-Feb. 1921 Geb. Saloniki/Griechenland, 13. Aug. 1867 (nach griech.-orthodoxem Kalender) Gest. Wien, 17. März 1940

Dr. Edwin Versbach-Hadamar GK 10. März 1921-19. Okt. 1925 Geb. Brünn, 17. Okt. 1883 Gest. Wien, 19. Dez. 1972 
Emmerich Herzfeld

GK

Geb. Smyrna/Türkei, 15. Juli 1880

Gest. Wien, 19. Okt. 1941

Heinrich Wagner

Geb. Wien, 8. Sept. 1865

Gest.?

Dr. Richard Steidle

GK

GK

Geb. Untermais bei Meran/Tirol, 20. Sept. 1881

Gest. KZ Buchenwald, 30. Aug. 1940

Otto Hausleitner

GK

Geb. Wien, 20. März 1902

Gest. Wiener Neustadt, 18. Juni 1968

Dr. Johann Manz

GK

Geb. Parndorf/Bgld., 31. Okt. 1918

Gest. Klagenfurt, 21. Nov. 1993

Dr. Manfred Schullern

GK

Geb. Wien, 8. Juli 1893

Gest. Innsbruck, 10. Sept. 1959

Dr. Paul Zedtwitz

GK

Geb. Wien, 17. Dez. 1911

Gest. Attersee, 14. Juni 1996

Dr. Georg Roessler

GK

Geb. Wien, 25. April 1913

Gest. Wien, 2. Okt. 1987

Dr. Ernst Hessenberger

GK

Geb. Gmunden, 11. April 1922

Gest. Wien, 13. Feb. 1975

Dr. Otto Fries

GK

Geb. Inzersdorf/NÖ, 15. Sept. 1904

Gest. Wien, 29. April 1981

Dr. Paul Wetzler

GK

Geb. Winterthur/Schweiz, 5. Nov. 1905

Gest. Wien, 26. Juni 1988

Dr. Heinrich Blechner

GK

Geb. Wien, 21. Juli 1929

Dr. Franz Pernegger

GK

Geb. Wels, 2. Juni 1931

Dr. Peter Klein

Geb. Wien, 4. Juli 1934

GK

GK
4. Jan. 1932-18. Juni 1934

7. Mai 1928-31. Dez. 1931

18. Juni 1934-13. März 1938

8. April 1948-25. Juni 1949

21. Juni 1949-8. Juli 1953

25. Aug. 1953-15. Juli 1954

15. Juli 1954-18. Mai 1956

20. April 1959-18. Dez. 1960

21. Dez. 1960-30. März 1965

15. Sept. 1965-28. Nov. 1969

14. Jan. 1970-14. April 1971

29. Juni 1971-15. Jan. 1976

21. Juni 1976-31. Aug. 1982

31. Aug. 1982-20. Feb. 1988

11. Sept. 1988-22. Sept. 1992 
Dr. Ingo Mussi

2. Okt. 1992-28. Aug. 1997

Geb. Darmstadt/Deutschland, 28. Aug. 1935

Mag. Arthur Schuschnigg

GK

13. Nov. 1997-31. Dez. 2000

Geb. Wien, 2. Juni 1935

Dr. Hans Sabaditsch

GK

15. Feb. 2001-27. Feb. 2003

Geb. Wien, 3. April 1941

Aussenhandelasstelle Triest - Die Leiter seit 1920

Wilhelm (Graf) Pace

1. Jan. 1948-17. Mai 1957

Geb. Deutschlandsberg, 30. Nov. 1896

Gest. Triest, 17. Mai 1957

Egon Metzel

1. Okt. 1957-30. Sept. 1962

Geb. Wien, 11. Jan. 1920

Dr. Rudolf Altenburger

16. Okt. 1962-23. Juni 1968

Geb. Salzburg, 7. Mai 1906

Gest. Triest, 23. Juni 1968

Dr. Ekkehard Strasser

Geb. Innsbruck, 2. Juni 1926

16. März 1969-13. April 1979

Gest. Zirl/Tirol, 12. März 1999

Franz Filli

Geb. Klagenfurt, 24. Juli 1922

1. April 1979-31. Okt. 1984

Dr. Herbert Geissler

1. Jan. 1984-16. Sept. 1990

Geb. Altschlaining/Bgld., 12. Juni 1940

1. Sept. 1990-31. März 1996

Dkfm. Stefan Wratschko

Geb. St. Leonhard, 4. Mai 1932 